Indian Journal of Science and Technology

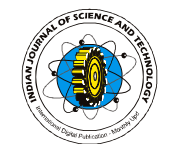

\title{
Determination of elastic constants of aluminium using laser based ultrasonics
}

\author{
T. Pramila, Anita Shukla, N.N. Kishore ${ }^{1}$ and V. Raghuram ${ }^{1}$ \\ Dept. of Physics, Christ Church College, Kanpur; ${ }^{1}$ Indian Institute of Technology Kanpur, Kanpur, India \\ pramilatabeti@yahoo.co.uk
}

\begin{abstract}
This paper deals with the determination of the elastic constants of aluminium from the analysis of laser generated ultrasonic bulk waves. A pulsed Nd:YAG laser $(1064 \mathrm{~nm})$ is used for ultrasonic generation in a thick stepped $\mathrm{Al}$ sample and a He-Ne laser is used for heterodyne detection of the generated signals. Ultrasonic signals obtained at epicenter and at off-epicenter position of the detection points are analyzed using wavelet transforms. Here the identification and of pressure as well as shear waves and the estimation of their velocities are done successfully. From the estimated velocities the elastic constants (Young's modulus, shear modulus, bulk modulus, Lames constant and Poisson's ratio) are calculated. The agreement of these constants with the standard values confirms the identification of shear waves at off-epicenter position. The work presented in this paper brings out the applicability of the study of laser generated bulk waves for the determination of elastic constants of any bulk material.
\end{abstract}

Keywords: Laser based ultrasonics, wavelet transforms, pressure waves, shear waves, elastic constants, Al.

\section{Introduction}

Laser Based Ultrasonics (LBU) plays an important role in material characterization. It is one of the well known Non Destructive Evaluation (NDE) techniques widely used for material characterization without detrimental effects on the structure being examined. LBU generation affords an opportunity to make truly noncontact studies in materials, in materials at elevated temperatures, in corrosive and other hostile environments. Lasers are able to produce various types of ultrasonic waves like pressure waves, shear waves, Rayleigh waves etc. simultaneously in bulk materials. Scruby and Drain provide an elaborated review on the principles underlying generation and reception processes of all techniques used (Scruby \& Drain, 1990). Monchalin presents a detailed discussion of different techniques with reference to exploitation of the power of the laser ultrasonic generation (Monchalin, 1986). Considerable work has been made to deal with techniques of measuring the wave speeds of different waves in conventional mode, and hence characterizing the flaws in materials (Krautkramer \& Krautkramer,1990) viz. simultaneous pulsed laser generation of elastic waves of different types in laminated composite materials (Corbel et al.,1993), laser interferometer detection and the determination of the theoretical displacement fields (Castegnede et al.,1994) and modeling of the ablation source in laser ultrasonics (Hoffman \& Arnold,2000).
Legendre et al. (2001) proposed wavelet-based method to perform the analysis of ultrasonic signals. By combining the time domain and the classical Fourier analysis, the wavelet transform provides simultaneous spectral representation and temporal behavior of the signal decomposition components. Monchalin et al. (1986) provides a good presentation about laser ultrasonic instrumentation. Daubechies (1992) has done signal analysis using wavelet transforms with different mother wavelets. Recent studies using LBU include generation of Lamb and bulk wave signals, which are analyzed using wavelet transforms (Manu Singhal et al., 2006; Pramila et al., 2007b; Pramila et al., 2007b). Present work is focused on generation, epicenter and offepicenter heterodyne detection and characterization of bulk waves generated in a thick stepped sample of aluminum. Signal analysis is carried out using wavelet transforms. The wavelet transforms are generated using MATLAB software. Results thus obtained are used for the estimation of elastic constants.

\section{Experimental details \\ Experimental setup}

The schematic layout of experimental setup is shown in Fig.1. A pulsed Nd: YAG laser is used to generate ultrasonic waves in a stepped sample of aluminum. The $1064 \mathrm{~nm}$ laser line with $7 \mathrm{~ns}$ pulse width was used for signal generation. The laser energy used was $200 \mathrm{~mJ}$. A heterodyne laser Interferometer ( $\mathrm{He}-\mathrm{Ne}, 633 \mathrm{~nm}$ ) is used

Fig. 1. Experimental setup

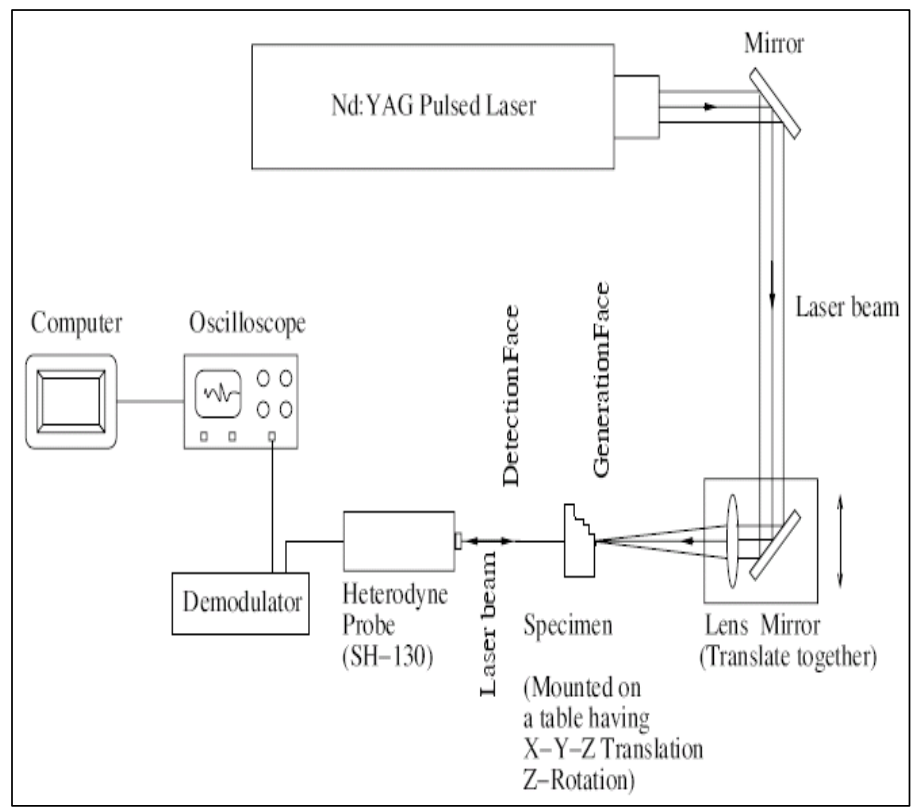


for signal detection. The signals are amplified and digitized using a digital oscilloscope. Recorded waveforms on the oscilloscope are transferred to a computer over an USB/Ethernet interface for subsequent storage and analysis.

The schematic representation of stepped sample is shown in Fig.2. The geometry of the sample is such that the centre point of each step lies on the arc of a circle. This is to ensure that time of flight of the bulk waves is constant for all angles of incidence. This geometry of the sample eliminates the need to take the attenuation effects into consideration while making calculations using signal intensities.

In the experimental arrangement using laser heterodyne detection, it is necessary that the probe laser beam should get back reflected. The heterodyne laser detects disturbances along the laser beam .If the ultrasonic waves generated in material are detected at 'epicenter'as shown in Fig. 3(a,), one can only detect the pressure wave displacements.

However, if the ultrasonic signal is picked up at offepicenter position as shown in Fig. 3(b) with the probe beam normal to the surface, the heterodyne will be able to pick up the components of vibrational amplitudes of both pressure wave and shear (horizontal) wave. transform. Wavelet is a short wave. A wavelet is a waveform of effectively limited duration that has an average value of zero. Comparing wavelets with sine waves, sinusoids are smooth and predictable whereas wavelets tend to be irregular and asymmetric. Wavelet analysis is the breaking up of a signal into shifted and scaled versions of the original (or mother) wavelet. Mother wavelet is a continuous function in both time and frequency and serves as a source function. Using wavelet analysis signal can be characterized in both time domain and frequency domain simultaneously. The wavelet transform is a

3-d plot of sample vs. scale vs. intensity. From the value of samples plotted on the Xaxis the time can be calculated while the frequency can be obtained from the value of the scale plotted on Y-axis and the intensity is depicted on Z-axis. The 3-d plot can be converted into 2-d plot by depicting the intensity variation with the help of various color schemes. In the present work the color code is such that the wavelet coefficients in blue represent the minimum value and those in red represent the maximum value. The red regions signify a strong presence of a particular frequency at that particular time.

Results and discussion

Recorded ultrasonic signals are analyzed using wavelet transforms. The details of the analysis are given in the following subsections.

\section{A. Wavelet transforms}

The FFT (Fast Fourier Transform) is a tool widely used for many scientific purposes, where one is interested in the frequency content of the signal .However, the information regarding the instantaneous intensity of a particular frequency component of the signal can not be obtained using FFT. To enable one to extract information regarding intensities vs. frequency vs. time behavior of any signal, Morlet introduced the wavelet
Fig.3. Ultrasonic wave detection (a) at epicenter and (b) off-epicenter

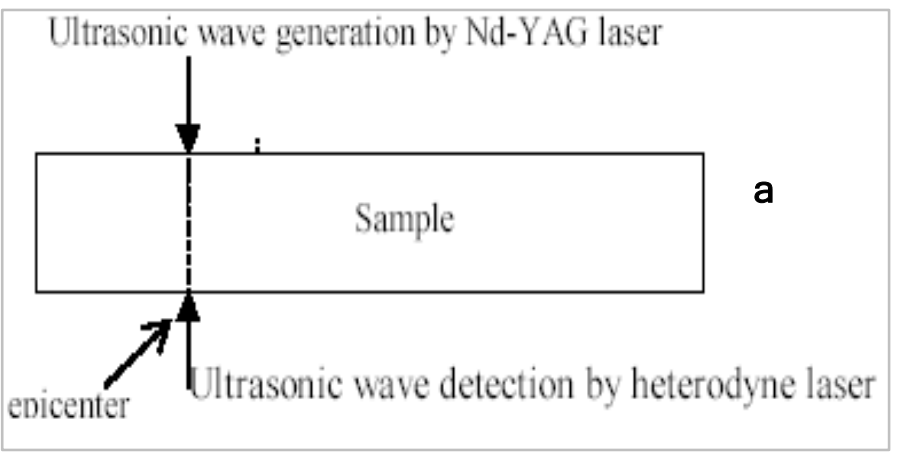

Ultrasonic wave generation by Nd-YAG laser

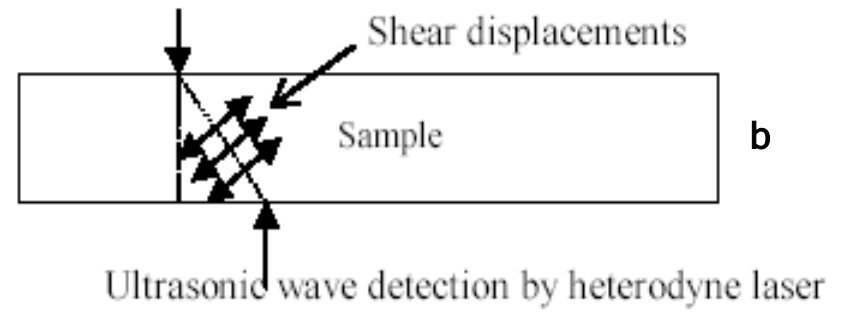

B. Recorded signals and wavelet transforms

Wavelet transforms for LBU signals of $\mathrm{Al}$ at $0^{0}$ and $40^{\circ}$ angle of detection are generated. The recorded signal along with its wavelet transform at $0^{0}$ And $40^{\circ}$ angles of detection are shown in Fig. 4 and 5 respectively. In these signals (a) gives the actual recorded signal (b) gives the wavelet transforms and (c) gives the coefficient line corresponding to particular frequency. This coefficient line gives the temporal behavior of a particular frequency component of the signal.
"Laser-based ultrasonics" http://www.indjst.org
Pramila et al. Indian J.Sci.Technol. 
Fig.4. The continuous wavelet transform of the ultrasonic signal at $0^{\circ}$ angle of detection corresponding to the frequency $645 \mathrm{~K} \mathrm{~Hz}$.

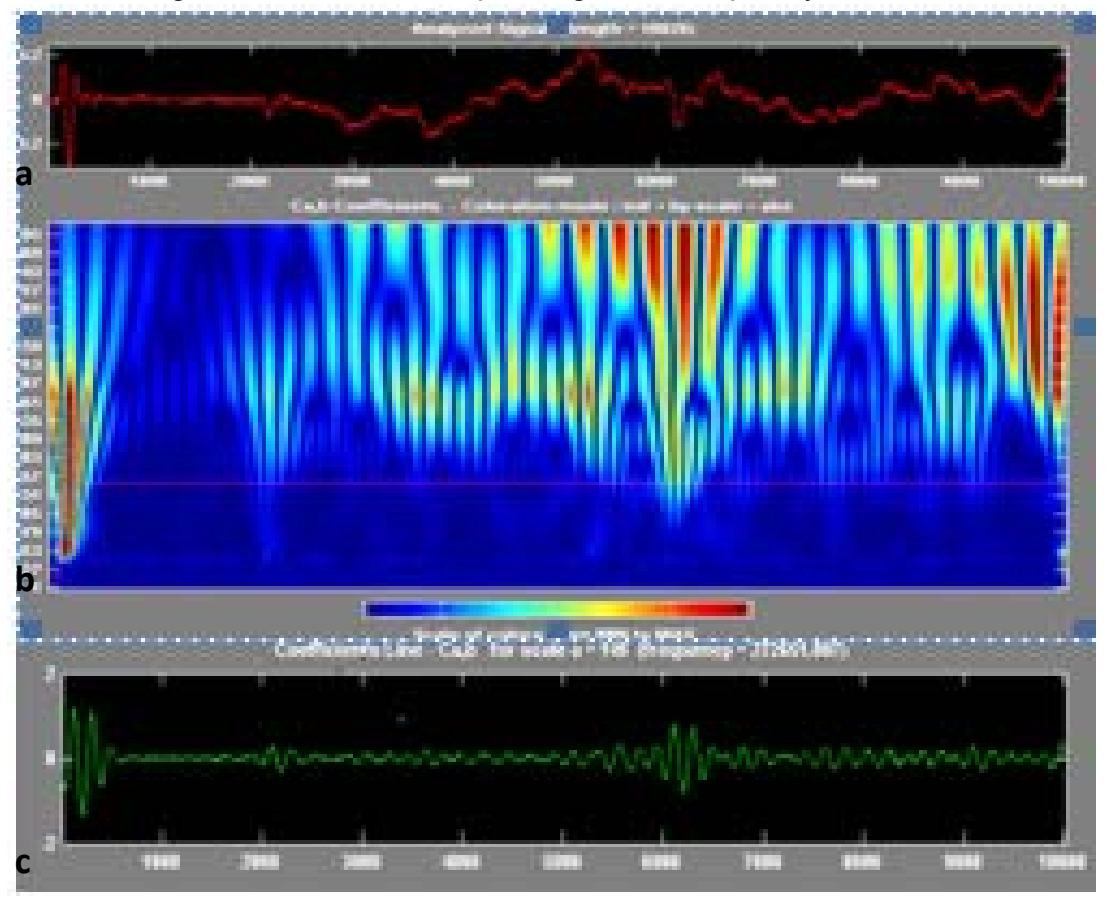

Vol.2 No. 12 (Dec. 2009)

ISSN: 0974- 6846

Al where the pressure wave has minimum intensity. Moreover, the time of arrival for this frequency band matches neither with first arrival or the first back reflection of the pressure wave signal. In addition, its arrival time is almost twice the time of flight of pressure waves, which is known to be the case for shear wave in Al. So, one can assume this band of waves to be shear waves. From the value of time of flight and the thickness $(0.025 \mathrm{~m})$ of the sample the velocity of shear waves is calculated to be $2907 \mathrm{~m} / \mathrm{s}$.

\section{E. Determination of elastic constants}

Elastic constants (Shear modulus, Lames constant, Young's modulus, Bulk modulus, Poisson's ratio) which are the functions of ultrasonic velocities are estimated using the following data.

$$
\begin{aligned}
C_{1}=\text { Pressure wave velocity } & =6410 \mathrm{~m} / \mathrm{s} . \\
C_{2}=\text { Shear wave velocity } & =2907 \mathrm{~m} / \mathrm{s} . \\
D & =\text { Specific density of } A l=2.7 \\
K & =C_{1} / C_{2}=6410 / 2907=2.2
\end{aligned}
$$

With the help of constants calculated and

\section{Epicentral detection (pressure wave velocity estimation)}

The LBU signal at epicenter i.e. $0^{0}$ angle of detection are recorded repeatedly and their wavelet transforms are obtained. A typical wavelet transform is shown in Fig. 4. In this wavelet transform pressure waves can be identified as the first arriving group of frequencies. From the wavelet transforms the coefficient lines for various frequency components are obtained. The arrival times of each frequency component are estimated. Using the sample thickness $(0.025 \mathrm{~m})$ and the times of travel the average velocity of the pressure wave is estimated to be $6410 \mathrm{~m} / \mathrm{s}$. This value is in excellent agreement with the known standard pressure wave velocity available in the literature.

\section{Off-epicentral detection (shear wave velocity estimation)}

As explained previously, if the detection is made at off-epicenter, with the probe beam normal to the surface, the heterodyne will be able to pick up the components of vibrational amplitudes of both pressure wave and shear wave as shown in Fig. 3(b). The heterodyne detection eliminates the epicentral detection of shear waves due to the fact that the particle disturbances are at transverse direction to the direction of detection.

In Fig. 5 from wavelet transform of the LBU signal at $40^{\circ}$ one can see the presence of a frequency band whose time of travel is 8.6 micro sec. This angle of detection is the critical angle for Research article OIndian Society for Education and Environment (iSee)
Fig.5. The continuous wavelet transform of the ultrasonic signal at $40^{\circ}$ angle of detection corresponding to the frequency $535 \mathrm{~K} \mathrm{~Hz}$

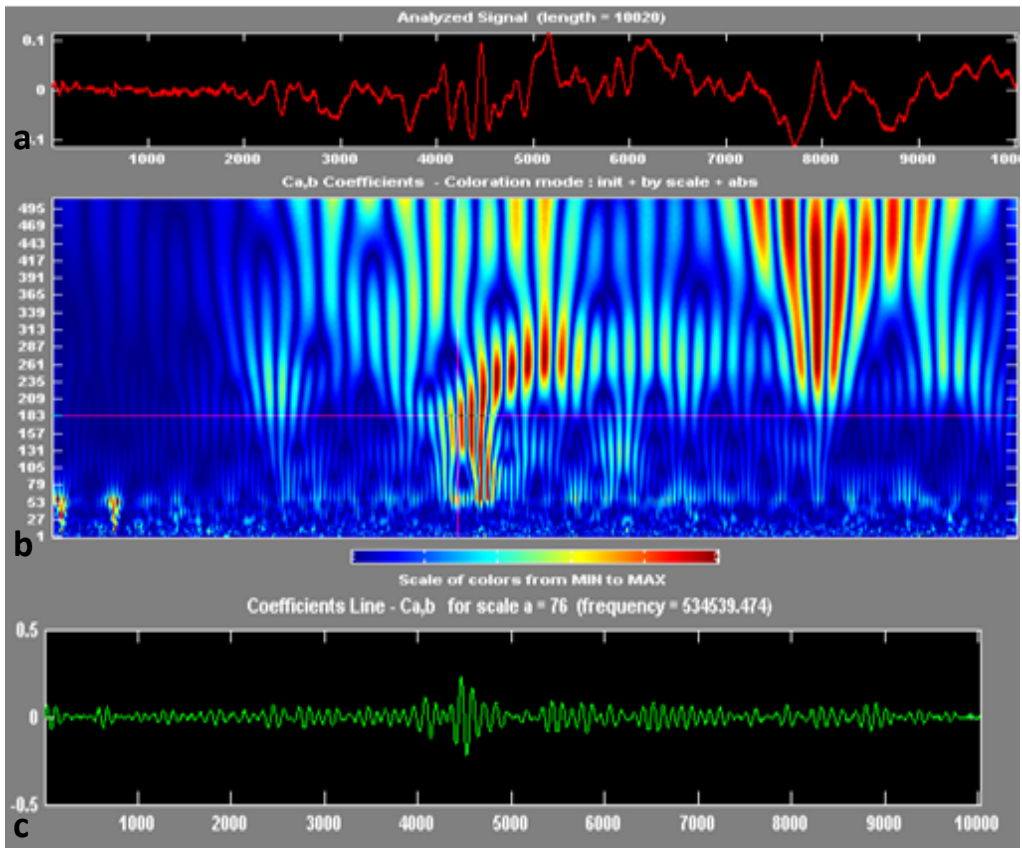

"Laser-based ultrasonics" http://www.indjst.org
Pramila et al. Indian J.Sci.Technol. 
Table 1 Elastic constants of aluminium

\begin{tabular}{|l|}
\hline \multicolumn{1}{|c|}{$\begin{array}{c}\text { Name of elastic } \\
\text { constant }\end{array}$} \\
\hline Shear modulus
\end{tabular}

\section{Conclusions}

In the present paper results of wavelet analysis of LBU signals obtained for stepped sample of $\mathrm{Al}$ are presented. Identification of pressure as well as shear waves and their velocity estimation is done successfully. The good agreement between estimated and standard values of elastic constants confirms the identification of shear waves at off-epicentral position. This work brings out the applicability of LBU technique where various types of bulk waves are simultaneously generated, for material characterization.

\section{References}

1. Castegnede B, Marc Deschamps, Eric Mottay and Andre Mourad (1994) Laser impact generation of ultrasound in composite materials. Acta Acoustica. 2, 83-93.

2. Christine Corbel, Franck Guillois, Daniel Royer, Mathias A. Fink and Renede Mol (1993) Laser generated elastic waves In carbon epoxy composite. IEEE Trans. on UFFC. 40 (6) 710-716.

3. Daubechies I (1992) Ten lectures on wavelets. Soc. for Industrial \& Applied Mathematics, Philadelphia, 199-209.

4. Hoffman and Arnold (2000) Modeling of the ablation source in laser ultrasonic. In: Review of Progress in Quantitative N. D. Evaluation. pp: 279-286.

5. Krautkramer J and Krautkramer H (1990) Ultrasonic testing of materials. 4th Ed., Springer-Verlag, New York.

6. Legendre S, Goyette J and Massicotte D (2001) Ultrasonic NDE of composite material structures using wavelet coefficients. NDT \& E Intl. 34 (1), 3137.

7. Manu Singhal, Pramila $T$, Raghuram $V$ and Kishore NN (2006) Characterization of laser generated bulk waves using wavelet transforms and pattern recognition. Asia Pacific Conf. on NDT, Auckland, Newzealand.

8. Monchalin JP (1986) Optical detection of ultrasound IEEE Trans. UFFC. 33, 485.

9. Monchelin JP (1986) Optical detection of ultrasound. IEEE Trans. on UFFC. 33 (5), 485-499.

10. Pramila T, Anita Shukla, Kishore NN and Raghuram V (2007a) Frequency analysis of laser-generated
11. Pramila T, Rashmi Shukla, Kishore NN and Raghuram V (2007b) A study of the spectral behavior of laser generated lamb waves using wavelet transforms. HSNT Conf., Chania, Greece. (October)

12. Scrubby CB and Drain LE (1990) Laser ultrasonic: techniques and applications. Adam Halter Bristol, Philadelphia, New York.
Pramila et al. Indian J.Sci.Technol. 